\title{
Digital Platforms as Sources for Organizational and Strategic Transformation: A Case Study of the Midblue Project
}

\author{
Andrea Resca ${ }^{1}$, Stefano $\mathrm{Za}^{2}$, Paolo Spagnoletti ${ }^{3}$ \\ Luiss Guido Carli University, Centre of Research in Information Systems, Rome, Italy, ${ }^{1}$ aresca@luiss.it, ${ }^{2}$ sza@luiss.it, \\ 3pspagnoletti@luiss.it
}

Received 16 August 2012; received in revised form 18 March 2013; accepted 9 April 2013

\begin{abstract}
The paper is centered on the role of technology and specifically of IT in the recombination of the factors of production. Particularly, the objective is to see how this technology contributes to the formulation of business strategies, the outlining of organizational structures and the alignment with the business environment. A theoretical framework is proposed that combines three perspectives. The first perspective stresses the importance of digital architectures in the modalities through which firms organize for innovation. Second, organization as a platform outlines a scenario for generating multiple organizational forms based on recombination of existent resources, routines and transactions. Third, digital platforms are seen as determinant tools for a dematerialization process that consents to recombine the factors of production for achieving a competitive advantage. The Midblue project provides an illustrative example of how strategy, structure and information technology co-evolve in organizations and how digital platforms can trigger transformation at both strategic and organizational levels.
\end{abstract}

Keywords: Platform, Digital product, Dematerialization process, Infrastructure, Ecogenesis 


\section{Introduction}

Traditionally, entrepreneurship is the activity related to the combination of the factors of production (land, labour, physical, human and financial capital) in order to produce goods or services for a market. The organizations support this combination. They represent the whole of roles and relationship (structure) led by a management able to follow a strategy that establishes an effective alignment with the environment [22]. The maintenance of this alignment is an extremely complex task because organizations and business environments continuously evolve. Strategy and structure are the main instruments through which organizations deal with this situation. Strategy is the plan of action to achieve a competitive advantage and structure represents patterns and relationships of entities that allow the accomplishment of this advantage.

Since Chandler's seminal work [11], the relationship between strategy and structure has been object of a large research activity in management studies. In organization studies, contingency theory [18] has a long tradition for studying the organizational structure/environment relationship. In order to be competitive, a strategy is formulated, an appropriate structure is envisaged for following it, and Information Technology (IT) is implemented to support both internal processes and actions in the competitive environment.

However, the question is if these policies, such as the strategy/structure alignment, the structure/environment alignment, and the strategy/IT alignment are still valid in order to be competitive. To achieve these alignments is difficult and takes time. In contrast, the level of competition and technological development requires a continuous recombination of the factors of production according to organizational forms that are no more led by traditional approaches in management or organization studies [6].

The relationship between IT, organizational structure and strategy has been investigated in the strategic Information Systems research stream [7], [28]. Previous works have focused on the degree to which an IS must be strategically, structurally and informally aligned with the organization, in order to achieve organizational performance [5], [19]. As opposed to such traditional approaches, a focus on the relationship between new digital architectures and organizational transformation has been recently advocated [2]. Organizational transformation is intended as the process through which an organization is re-aligned with its environment.

New digital architectures are more flexible and scalable than pre-Internet ones [21] and therefore their transformative power is higher. Furthermore, since socio-technical, economic, and political trends enable new trajectories for transformation, organizational inertia is increasingly eroded [2]. For instance, organizations can take advantage from the modularity, scalability, and interoperability of Service Oriented Architectures for transforming their structure and implementing new strategies.

In this scenario, the objective is placed on the mechanisms through which new digital architectures enable organizational transformation. The points of reference used to investigate this innovation process are strategy, structure and environment.

The Midblue project is a project for the provision of multimedia contents to visitors of circumscribed areas related to what they are observing as in the case of museums, trading fairs, exhibitions etc. It is the case study that was selected in order to investigate these issues. Due to the passage of time and environmental changes a two phase project emerged leading to the design of two different IT artifacts defined as Midblue version 1 (Mv1) and Midblue version 2 (Mv2). Although the two solutions share the common goal of adding new capabilities in the provision of services and contents, the followed strategy, the organizational structure adopted and the interpretation of the environment differ significantly. They show different levels of embeddedness in the structure and strategy of the organization adopting the solution and the comparison of these two artifacts provides an illustrative example of the organizational transformation process showing the environment re-alignment at both strategic and structural levels.

The first section is dedicated to build a conceptual framework for analyzing the Midblue project. Three theoretical perspectives are integrated in this framework, which are related to the IT architecture, the structure, and the strategic levels. The research methodology is then presented together with the case description. A discussion section applies the conceptual framework for analyzing the Midblue case summarizing the findings and with the conclusion section the paper comes to an end.

\section{Theoretical Framework}

In order to build an integrated interpretative framework, three perspectives are considered. The first perspective stresses the importance of the technological architecture in the modalities through which firms organize for innovation [37]. The organization as a platform [6], [8] is the second perspective taken into consideration. Finally, dynamics of value creation are at the basis of the third perspective [24]. Technology and particularly IT are, here, seen as drivers and tools for a dematerialization process that allows to recombine the factors of production for 
achieving a competitive advantage. Table 1 summarizes the main assumptions of each perspective and provides a list of sources on which our theoretical framework is grounded.

Table 1: Integrated framework for IT enabled organizational transformation

\begin{tabular}{|l|l|l|}
\hline Perspectives & \multicolumn{1}{|c|}{ Key concepts } & Main sources \\
\hline IT architecture & $\begin{array}{l}\text { - Modularity and standardized interfaces } \\
\text { - The layered modular architecture of digital products } \\
\text { - Products as platforms } \\
\text { - Border control }\end{array}$ & [3], [14], [37] \\
\hline $\begin{array}{l}\text { Organizational } \\
\text { Structure }\end{array}$ & $\begin{array}{l}\text { - Management of uncertainties } \\
\text { - Technological bet } \\
\text { - Ourfing the business environment }\end{array}$ & [6], [8], [30], [35] \\
\hline $\begin{array}{l}\text { Business } \\
\text { Strategy }\end{array}$ & $\begin{array}{l}\text { - Lalue constellation } \\
\text { - Ecogification, unbundleability, and rebundleability } \\
\text { - Upframing as a business strategy }\end{array}$ & [15], [24]-[26] \\
\hline
\end{tabular}

\subsection{The Layered Modular Architecture of Digital Platforms}

Digital products are characterized by specific architectures that defines them [37]. At first the term architecture requires further specifications. Each product distinguishes by a specific architecture that outlines modalities through which its parts and functions are composed. For example, the integrated architecture and the modular architecture represent opposite visions through which to design goods. In the first case, functional elements and physical components are closely interlinked. This leads to a high performance of the system in question. If a product is designed according to a modular architecture, however, the functionalities are not only related to specific physical components. It means that they are recombinable giving rise to different forms of the product. In this case, the role of the interface between a component and the other becomes essential and standardized.

Digital products are also characterized by a layered structure in which elements of different nature are combined at different levels and thus a layered modular architecture represents them [14]. Basically, these products are composed by four layers: devices, networks, services and content. The devices can be further subdivided into the physical aspects (i.e. hardware) and what makes their control (i.e. firmware) as well as the other layers. The network can be divided in its physical infrastructure (i.e. cables, etc.) and the protocols managing the transmission (i.e. TCP / IP). Content creation and its editing, storage and consume is provided by the service layer. The content layer is represented by texts, sounds, images and video for use or consume. Among the contents, metadata are considered as well. A tablet is a typical example in this respect. It is a device connected to a network for providing functionalities to manage contents and allow their consumption or use.

However, an increasing number of products are characterized by the presence within them of digital components (i.e. cars, household electrical appliances etc.). This contributes to imposing the so-called layered modular architecture [14]. It is a hybrid between a modular architecture and a layered architecture. In this instance, the product is formed by components according to a specific functional hierarchy, so that they nested and fixed, and by components not necessarily embodied in a single product. These components are considered as product agnostic [37]. Let's think about Google Maps. It can be conceived as a standalone product but also as a component of a website, a smartphone, a car etc. These digital products can be enacted in different ways and be part of different design hierarchies. This means that it is not possible to know in advance whether they will be used as standalone tools or as modules in a given layered architecture.

A product of this kind cannot lead to differences in degree as in the case of the modular architecture but to differences in type. The example of the digital camera can be significant in this respect. It can be used as a video player, internet client etc. [14]. Thus, digital products characterized by layered modular architectures remain fluid, and constantly evolving. However, unlike the pure layered architecture, some limitations are imposed by the physical components of the product (a camera is still a camera).

A digital product characterized by a layered modular architecture can be both a platform and a product. The iPad is a product. However, other firms may invent some new applications or peripheral accessories to expand its capabilities. In this way, companies that manufacture products with this architecture invest also in these products as platforms considering different markets and the possibility to build innovative product ecosystems around them [14]. As a platform, its layers can become source of further digital products as different players can take advantage of it contributing to its continuous innovation [3].

These dynamics lead to a market in which companies may compete at one layer and collaborate in another layer. Besides, the objective is to attract companies able to create those conditions that give the possibility to be present in 
layers that are outside the digital product seen as a platform. Therefore, the importance to design platforms that attract a large number of heterogeneous components and that belong to different design hierarchies is stressed. Hardly a single firm because of economic, structural, cognitive, and institutional constraints can be generative. The presence of an organizational logic that involves heterogeneous players pursuing their own strategy of innovation is fundamental. These players are not limited to firms in the same sector but also across sectors.

This context, due to the presence of digital products characterized by a layered modular architecture, is represented by a doubly distributed network. A network is distributed because value creation lies in the generativity based on the combination of heterogeneous resources present at different layers and is double because 1) the control over the components of these products is distributed across several businesses and 2) knowledge related to these products is distributed among diverse disciplines and communities. With these premises, what is crucial is the capacity to design a digital product platform to mobilize and inspire a doubly distributed network. In this respect, to attribute new meanings to potential products and services constantly redefining their boundaries through a reformulation of the product ecology is determinant [17], [32].

This scenario leads to several challenges in strategic research. At first, the digitization of products blends the boundaries between products and between industries. Google and Apple are a classic example in this context. Then, the layered modular architecture provides a range of possibilities to insert digital components into a physical product representing strategic choices to pursue digital innovation. A further challenge lies in the fact that having at disposal platforms of digital products is fundamental to control or not the key components or specific combinations of components within certain layers. This control can lead to a competitive advantage. In particular, businesses must continually wonder: a) what should remain open and closed in the digital product platform; b) how to identify and monitor key components that are of strategic importance; c) how to build efficient incentives for alluring companies to invest in the product platform. Again, layered modular products are conceived as a platform and a component at the same time. However not all businesses can follow this strategy simultaneously. For example, it is expected that a small start-up will focus on components until it reaches a stable number of users across multiple platforms. Finally, considering that the environment in which these businesses operated can be represented by the doubly distributed network, it is crucial to be attractive to companies that are heterogeneous and far from the business. For this proposal, a strategic resource is the border control both the technological one (e.g. APIs and SDKs) and the social one (i.e. incentives, property rights and forms of control) [13].

\subsection{Organization as a Platform}

In a scenario characterized by technological innovation, intense market competition, and a continuously evolving business environment, the capacity to change and to manage uncertainties is fundamental. But what does it actually mean to change and to manage uncertainties? What principles can be followed in order to combine the factors of production in order to be competitive? To stake on a specific technological paradigm and to commit to its development is seen as a possible solution in this respect [6], [8]. The technology at stake can become what envisages the business strategy to be followed. It outlines a setting in which it is possible to figure out the growth of a specific business. Besides, the possible evolution of a specific technology is seen not only as a way to define a strategy but also as a factor that outlines a specific organizational structure. In other words, the combination of procedures and transactions of an organization can be preconfigured by the characteristics of the technology [16].

If a new generation of technologies establishes itself, a series of important consequences can emerge. Products, all of a sudden, are outdated and the same destiny can involve their assembly lines and production processes. An entire culture (mental models, skills, experiences, traditions) [12], [29] and also the same organization identity have to be abandoned as related to an obsolete technology. Nevertheless, we cannot expect that organizations are able to make such a quick shift so that abruptly they embrace the new technology completely. This process takes time and probably it does not make sense to have this sort of objective as a new technological system can follow in a relatively short time. In this situation, organizations are the results of the sedimentation of structures, processes, routines and transactions that have established themselves in the course of time. There are not the conditions to select an appropriate organizational form according to transactions or coordination costs. This process takes time and the risk is to lose momentum facing a surprise or a technological wave. In this respect, one of the most important management assets is the capacity to deal with these circumstances. Therefore the strategy and structure alignment loses its relevance. It is not a priority to follow the alignment when the solution is rather to seek flexibility to surf this business environment [20], [35].

To figure out an organizational form that instead of addressing the reduction of transaction and coordination costs or its alignment with the strategy, is able to promote flexibility, the management of surprises and new technological trajectories is at stake now. The concept of platform is proposed in this respect [6]. Actually, it is a metaorganizational form. In fact, the question here is not to propose a specific configuration of roles and relationships or a further form for governing transactions but to work out what to do when a new technological trend is considered promising or other surprises completely change the business scenario.

The digital architectures discussed so far evoke the concept of platform here presented. Specifically in computer firms, the computer platform becomes the point of reference for the developments of new products. And, at the same time, it traces back to the organizational structure. In some sense, strategy and structure are recombined in the 
metaphor of the technological platform. Therefore, departments, functions and divisions can be represented by this metaphor. It goes without saying that this becomes a powerful theoretical framework for managing business situations described above. Let's consider, for example, the PC/386 platform. Its motherboard, its microprocessor etc. outline a series of products with different characteristics according to the components made available by the platform. However, at the same time, it envisages a scenario of supplier and customers and the organizational structure able to exploit it. With the PC/486 platform things change as a new configuration of products, suppliers, customers and organizational structures becomes possible. The objective is to combine the factors of production in order to take advantage of this further platform. In these situations market leaders tend to be temporary. A new technological paradigm represents an opportunity for new entries able to envisage innovative businesses and how to respond to them.

As a technological platform, the platform as an organization can be conceived as an arrangement able to provide a series of solutions dealing with crisis affecting not only organizational strategy and structure but also organizational culture and identity [6], [8]. What are the characteristics of this arrangement? Why does it represent a solution in order to deal with these situations?

At first, to conceive it as an organizational structure that leads to a reduction of coordination costs or organization costs is inappropriate. At stake there can be a technological trajectory or another primary task that inevitably affect factors such as culture and identity. Here, the efficiency of an organizational structure is pushed into the background. Second, it is in the long term that features of this arrangement come out rather than in a specific point in time. Only according to a longitudinal perspective is it possible to observe the sequence of organizational forms that follow one another and mainly the speed and friction of these passages. The management leads them. A management that tends to bet to a specific primary task (technological trajectory, environment change etc.) and, in consequence of this bet, alliances, mergers and acquisitions, vertical integration etc. do follow. The organization as a platform, due to its characteristics, supports the recombination of the factors of production and then this sort of betting. This activity is far to be planned. On the contrary, it is opportunistic and based on improvisation but is not accidental. The platform is like a chameleon, it continues to reformulate its assets in order to deal with the next bet. These assets are already available. They are not the result of a planned activity. Rather, what is at hand such as routines and pre-existent structures is re-used for a new endeavor as in the case of open source software adoption [30]. Therefore, at a first look, a hierarchy or a matrix emerge but as an effect of a meta-level constituted by the platform. The platform is seen as a virtual and collective cognitive scheme enacted to deal with market contingencies, a technological innovation or competitors' moves.

The main characteristics of the organization as a platform can be summarized as follows: i) it provides an organizational architecture based on a multiplicity of structures; ii) frames and routines can be easily transformed across diverse organizational settings; iii) it provides a context in which available resources can be reinterpreted in innovative ways reallocating them in completely different frames; iv) superficially it acquires a common configuration but actually manifests itself facing surprises and using resources in an unexpected way; v) it achieves flexibility outlining structures that provide innovative answers to chaotic events.

\subsection{Ecogenesis as a Business Strategy}

The role of IT for entrepreneurship is seen according to Normann's view [24]. This perspective focuses mainly on strategy formulation. In other words, how technology innovation and IT lead to the envisaging of novel business strategies. Particularly, the point is to see how value is created. Value creation is the results of the establishment of a series of relationships with a range of players that rally round a specific company. The sum of the value provided by these relationships allows to achieve a competitive advantage and then to the possibility to align the strategy with the environment. The term value constellation is used in this regard [1], [4]. In contrast with the value chain [9], where value is added from each stage of production (from providers to customers of a specific business unit), value constellation emerges from the relationships established with suppliers, customers, government, universities etc. Underlying the establishment of these relationships, there is the overcoming of some constraints represented by time, place and by the actors with which to intertwine relations and the possible configurations of these. Therefore, the question is when and where activities can be done, who is able to perform them and in collaboration with.

Technological innovation and, in particular, IT and the Internet have significantly influenced this process. A path that is substantiated by a process of dematerialization. At the basis of this process there are two phenomena in particular: liquification and unbundleability.

The liquification is determined by the separation of information from the physical world. For example, the same content can be sent by email or through postal services. In the latter case, the involvement of the physical world is much more important. The liquification, moreover, allows a kind of mobilization of information and its combination so that tremendous amount of new information can be created. Information that produce information which in turn produce information and so on [15]. The functioning of financial markets makes the idea of this phenomenon. However, the liquification process encounters an obstacle when the repository of a particular piece of information or knowledge is the subject as such. Tacit knowledge or knowledge as a direct emanation of a specific socio-cultural context is a good example. 
The unbundleability, instead, has to do with the possibility of reformulating the production activity according to time, the place where this is carried out and the involving actors. For example, due to e-commerce platforms, goods are available 24 hours a day, 365 days a year (time). Further, goods are not only available in traditional shops but also on the net (space) and customers can acquire a new role as in the case of lkea furniture where they are in charge of the assembling function rather than Ikea's employees (actor). To say it differently, unbundleability represents the possibility provided by IT to dissect activities of a specific business through innovative solutions.

Technological innovation and IT do not only support the dematerialization process but also the rebundleability process [24]. What was liquefied and unbundled can be re-bundled according to innovative forms. This means that economic activities can be reconstructed to take advantage of the most valuable assets available. A design vision emerges in which external actors and new competences are mobilized, old business borders are overcome and actors' roles are reshuffled. If this business reconfiguration involves not only products and services but a whole business system, an ecogenesis occurs. The rules of the game transform, leading to a new infrastructure and new business ideas that influence strategies, actions and networks of other actors within the system.

Normann [24] turns to the concept of prime mover for defining an economic actor able to reconfigure a business up to promote an ecogenesis. But what is a prime mover? It is not a question to take advantage of a technological innovation or of a market imperfection. The question is a mind-set of value creation and the term upframing is used in this respect. Upframing is considered an attitude toward a larger conception of value creation, the possibility to envisage a more articulated business system. Of course this is not only an intellectual exercise. The point is to follow this business model establishing an innovative reconfiguration bringing "players with desperate assets and competence together into forming a new, functioning value-creating system" [37] p. 66. The assumption, here, is that the organization structure faces difficulties to follow the strategy outlined and becomes decisive to mobilize coproducers, also on the basis of non-monetary exchanges, to collaborate to the realization of the new value system. Here the primary task is not only to be competent and efficient producing goods and services but to mobilize competences present in the environment and in the ability to establish new rules of the games that go beyond traditional business sectors and business systems. At the basis of this ecogenesis a series of factors has been identified: i) the importance of the concept of infrastructure as the metaphor for building a larger system able to influences strategies, actions and interactions of the players operating in it. An infrastructure that combines technological aspects and conceptions for creating scenarios to be actually realized; ii) a two phases process in which the infrastructure and its related network give, at first, shape to the conditions for supporting interactions and then a stable and progressive evolution of the system; iii) the importance to recognize and mobilize dormant resources as parts of a systemic vision through the infrastructure and other non-monetary resources of exchange (i.e. barter); iv) the exercise of power on other players is not considered relevant. Rather, win-win situations are important; v) to build a world is considered determinant. Elements of the system have to be aware of the rule of the games and of the design at the basis of the organization arrangement envisaged.

\section{Research Methodology}

The research object of this paper concerns the role of technology and specifically of IT in the strategic and structural organizational transformation. Particularly, the objective is to investigate how this technology contributes to the formulation of business strategies, the outlining of organizational structures and the alignment with the business environment.

Based on the theoretical framework developed above, we conducted a comparative case study to investigate how strategic and structural organizational transformations are linked to the architecture of IT artifacts. The purpose of the case study was to empirically confirm that the architecture of digital systems can trigger organizational transformation at both structural and strategic levels. Furthermore, we aim to show how different literature streams can be integrated for achieving a better understanding of the organizational transformation phenomenon.

Case studies provide rich descriptions of a phenomenon and also provide understanding by allowing in-depth analysis by researchers [33]. A single-case study with two embedded units of analysis has been conducted in the context of the Midblue project, whose goal was to enhance the capabilities of a service organization that manages content delivery in a circumscribed area (i.e. museums, trading fairs, exhibitions, archaeological sites etc.). In this context IT capabilities in supporting the exchange of multimedia contents and information, are the basic elements for building new IT solutions. They can transform such organizations and give them a competitive advantage.

Our primary source of data includes project documents, direct observation and focus groups with project participants and IS scholars. We used focus groups to corroborate and clarify the data collected through project documents. The use of multiple sources of information give help to prove the credibility and dependability of interpretive case study [36]. The project documents provided a first source of evidence for depicting the differences between Mv1 and Mv2. During meeting and seminars, some feedbacks were collected from project partners, practitioners and IS scholars. Data were analyzed and interpreted using the integrated framework for IT based organizational transformation [34].

The Midblue project provides an illustrative example of the phenomenon under investigation for different reasons. First, the project is relatively simple in terms of goals, project structure and stakeholders. The goal of the project was 
to implement a solution for transforming the means through which contents and information related to the objects that are observed by the customers during their visiting experience are provided. A Small and Medium Enterprise (SME), operating in the IT industry, was the key actor in the project with the scientific support of a research center. A local authority was funding the project in order to foster the innovation in the specific geographic area. Second, being members of the research center that was involved in the project, we had a privileged access to the Midblue information and stakeholders. Furthermore, having coordinated the design and evaluation activities of the project, we directly observed the phenomenon under investigation due to the opportunity to have complete access to a large set of project documentation. Third, the scope of Midblue has considerably changed during the project duration and it is interesting for revealing the evolutionary aspects of organizational transformation. This last aspect offers the possibility to perform a longitudinal case by specifying how certain conditions changed over time leading to the design of Mv1 and Mv2.

These three aspects are advantages that justify both the choice of a single-case study as a strategy for conducting the research and the selection of Mv1 and Mv2 as two embedded units of analysis for avoiding the unsuspected slippage problem [20] p. 50. In fact, the comparison of two different scenarios emerged during the project reveals itself rather interesting, despite one is the development of the other. The reasons at the basis of these differences are investigated in detail as sources of considerations on the role of these digital architectures, on the formulation of strategies and the outlining of organizational structures.

\subsection{The Midblue Case}

In the summer of 2009, the Lazio Region (Italy) issued a research grant dedicated to SMEs and research academic institutions (i.e. university, research centers etc.), for presenting proposals for innovative IT research projects. Such projects were requested to contribute to the development of one of regional strategic priorities such as aerospace, IT and multimedia, economics of cultural heritage, energy, and biotechnology. The spirit of this initiative was to allow the transfer of knowledge and competences from academic institutions to SMEs.

The main objective of the Midblue project was to design, implement and evaluate an IT system for providing multimedia information to visitors of circumscribed areas (i.e. museum, trading fair, exhibition, archaeological sites, etc.). Visitor are observed and the project objective was to enrich their visit experience. One of the peculiarities was the use of users personal devices (i.e. smart-phone, mobile phones, and other handled devices belonging to the visitor) without using any additional devices such as legacy audio/video guides.

The research center involved in the project provided its knowledge and competences for performing the following steps: i) formulate the state of the art about the use of IT for supporting the visiting of circumscribed areas adopted in the cultural heritage context; ii) investigate visitors' behaviors of the several channels used for providing contextual information (panel, audio-guide, totem, etc.); iii) recognize the potential actors involved in the new scenario (visitors, organization, etc.) and their needs; iv) design the IS; v) supervise the system implementation and its testing performed by the SME; vi) perform the evaluation of the implemented solution.

The first version of the Midblue system (Mv1) aimed to provide multimedia content distribution to users in a small and delimited geographic area (a museum, an archaeological area, a trading fair, an exhibition, etc.) using the Blue-tooth connection available at that time in almost all mobile personal devices (smart-phone, mobile phone, etc.). The choice to use the Blue-tooth connection as the channel for exchanging information was due to two main reasons. First many personal devices support this kind of connectivity. Second, the characteristics of this standard allow to recognize the device when it is close to the point of interest (POI) (maximum around 10 meters, even though, taking into account the signal strength, it is possible to consider a shorter distance).

The proposal was submitted in the summer of 2009 and in autumn of the same year the project was approved. The starting of the project activities was planned by the end of 2009 conditioned to the payment of the first part of the grant. Some problems in the funding local agency made it not possible to follow the initial planning and the actual beginning of the project was shifted month by month until autumn 2011. Almost three years following the writing of the project proposal.

After this period, the applicants decided to update the proposal by revising the project scope and objectives in order to consider the new environmental scenario particularly IT advancements. In 2011, a large portion of the potential users was equipped by a smart-phone with a WiFi connection in addition to the Blue-tooth one. Another important aspect that has been considered in this phase is the spread of the social network phenomenon (i.e. Facebook, Twitter, Google Plus, etc.) during the 2009-2011 period. In 2011, their usage was largely diffused, and users interact with social networks also through their personal mobile devices.

On this basis, a second version of the Midblue scenario (Mv2) was depicted. In particular, in Mv2: i) the Blue-tooth connection is only used to notice that a device (and hence its owner) is close to the POI; ii) in contrast with the past version, information is transmitted to visitors through the WiFi connection; iii) users have the possibility to share the content received (text, image or video) through their preferred social network, using an additional function on their devices. Also in this case the transmission is managed through WiFi in order to ensure the bandwidth for multimedia content. 
The project partners made an agreement with an organization that was interested in hosting the pilot phase, according to the new project plan, during the first quarter of 2012. The apes' area of a zoological garden, managed by a group of researchers interested in studying apes' behavior. Researchers who are periodically involved in field studies in remote areas where they collect data on the ape's behavior through direct observation. In this context, they played an important role in the Mv2 since they agreed to take part in the pilot study by providing contents (text, images and video) in a dynamic way.

During the first meeting with content providers, an additional requirement came to the fore: the willingness of such researchers to have the opportunity of disseminating the results of their studies by targeting seniors, kids and other classes of users outside the traditional range of scientific dissemination. Specifically, there was the possibility to circulate contents through social networks and to participate in online communities activities legitimating their role in the academia and in the society at large.

This possibility was transformed into the design of a new capability of the system for collecting instantly and automatically contents produced by online communities using external social network tools (i.e. Facebook pages). In this way content providers can interact using the functionalities of social network sites and share contents such as likes, posts, videos, pictures, etc. with people sharing the same interests (i.e. apes' behavior). Such contents are instantly and automatically transmitted to the visitors of a POI giving them the possibility to further circulate additional comments within their personal networks.

Now, two kinds of communities can be identified: visitors and scholars. Each visitor is a member of his/her personal network and can share contents provided by the system with his/her contacts. On the other hand, researchers are part of the collaborative communities of content producers that are studying apes' behaviors. In this way, the system enables an information flow only from the scholars' community to the visitors' communities, and not vice versa.

During the next round of requirement definition, a way for allowing a sort of interaction between the two communities has been proposed. For example, visitors can take a picture or a video with their smart-phones during their tour and through the system they can share this content with the researchers' communities. Researchers can evaluate it and eventually enrich the set of contents provided by the system. In this way the Mv2 blurs the boundaries of the two communities.

\section{Discussion}

A deep understanding of the organizational transformation process within the Midblue project can be achieved by applying the key concepts of our theoretical framework for performing a comparative analysis of Mv1 and Mv2 along architectural, structural, and strategic perspectives.

\subsection{Midblue as Digital Product}

The IT architecture perspective suggests to look at Mv1 and Mv2 as digital products [37]. At first, the analysis is about the architecture of these IT artifacts and specifically if they are built according to a modular architecture or a layered modular architecture. The former seems to characterize Mv1. In fact, its components are part of a single design architecture even though it can acquire several forms. Size and dimension can be adapted but necessarily they share a matching interface. In contrast, components of Mv2 are part of a different architecture. It goes without saying that social networks such as Facebook or YouTube can be integrated in a large number of products/platforms. The term assemblage [10], [23], [27] is considered appropriate to describe this platform identified by a layered modular architecture due to the fact that its components are part of different products/platforms.

Differences between Mv1 and Mv2 emerge clearly analyzing the layers (device, network, service, and content) of these digital products. The device layer (hardware and firmware) is composed by a series of components integrated by the Mv1 application. Its functions are circumscribed to the management of information flows linking the central server to a number of Blue-tooth stations that are located in the spots of interest within the zoological garden taken into consideration. Mv1 can be conceived as a sort of closed system that makes available an additional channel for distributing information from the organization to its customers. The characteristics of the network (physical infrastructure and transmission protocol) confirm this scenario. A Blue-tooth technology circumscribes to the establishment of personal area networks (PAN) imposing limits to the provision of services (content available only on-site) and contents.

On the other hand, Mv2 devices and networks are more articulated. In this case, the Mv2 application represents the system integrator for different components that include also social networks such as Facebook and YouTube. Here, the Blue-tooth technology is used only to geo-localize visitors while information exchange takes place through a WiFi channel. This shift in the technology transmission mode is enabled by the availability of visitors' personal devices equipped with a WiFi interface. Furthermore, the presence of smart devices in the Mv2 scenario entails makes possible the access to digital contents either on site or on social networks. In fact, the WiFi capability enables the 
internet connection and hence the possibility to create, store and edit contents (i.e. a monkey's picture) and to share them on social networks.

The fact that Mv1 is designed according to a modular architecture curbs its potential development. It is organization specific in the sense that its adoption is restricted to similar organizations (museums, archaeological sites, exhibitions, etc.). Mv2, being designed according to a layered modular architecture, is considered more generative even though it is an evolution of Mv1 [38]. Differently from the latter that supports only a Blue-tooth connection for content transmission, Mv2 combines both the Blue-tooth and WiFi connections and the social networks interface. Also, its configuration can be easily deployed in similar contexts but further configurations are not excluded given the role of visitors and researchers that can promote and implement innovative solutions.

The concept of doubly distributed network represents a further step for investigating the characteristics of Mv1 and Mv2. According to Yoo et al. [37] these networks are characterized by the presence of heterogeneous resources at different layers, by a components' control distributed across several businesses and by knowledge processes that encompass several disciplines and communities. Therefore, can Mv1 and Mv2 be considered a doubly distributed network? Actually, in Mv1, heterogeneous resources are present in different layers as suggested by the analysis of its devices, networks, services and content. However, the control of resources is centralized in the focal organization be it a zoo, a museum, an exhibition or an archaeological site. Also knowledge related to content is not distributed among different disciplines and communities but is aggregated in the focal organization. Considering Mv2, the scenario is different. In this case, heterogeneous resources are present as well. Nevertheless, the control of resources is not centralized but shared, eventually, with visitors and researchers. Besides, knowledge related to content is not circumscribed to the focal organization but it is created in a collaborative way by communities of visitors and researchers. This means that, differently from Mv1, Mv2 can be considered a doubly distributed network.

\subsection{Midblue as an Organizing Platform}

The analysis of Mv1 and Mv2 according to the perspective proposed by the organization as a platform [6], [8] poses some terminological problem. Mv1 and Mv2 are entities that provide contents and services whereas organization as a platform consists in a theoretical perspective for envisaging organizational solutions able to face market contingencies, technological innovations or competitors' moves.

The organization as a platform characterizes itself for its architecture based on a multiplicity of structures. Mv1 can be traced back to this architecture with difficulty. The organizational structure of the focal organization (zoo, museum, exhibition, archaeological site etc.) tends to prevail. In Mv2 the organizational structure is more articulated as users and researchers respective communities are added outlining an architecture composed by a multiplicity of structures. The organization as a platform is characterized also by the transformation of frames and routines across different organizational settings. In this case, Mv1 does not enable this transformation and supports only a single organizational setting. In contrast, in Mv2 content production and distribution can be easily transferred to users and researchers. Among the capabilities of the organization as a platform there is the possibility to reinterpret available resources such as skills, information, products, materials etc. according to innovative ways and to reallocate them in completely different frames. Mv1 does not show these characteristics. The reinterpretation of resources and their reallocation are limited by a context in which the focal organization plays a decisive role. The picture changes in Mv2. Here, actually, contents and services provided can be re-enacted due to the role of visitors, researchers and their respective communities and re-shaped by social networks such as YouTube and Facebook.

The point is to investigate the shift from an application supporting and automating existing processes and tasks (Mv1) to a technological platform that opens its boundaries to external communities of users (Mv2) [30]. What have been the main factors at the basis of this shift and which organizational configurations have taken place? In Mv2, the adoption of the WiFi technology for information transmission marks a significant change in comparison with Mv1. At the basis of this decision there was the intention to follow a new technological trajectory based on the diffusion of the WiFi capability in personal devices (i.e. smart-phones). However, the new system is not only the result of the adoption of the WiFi capability for complementing the geo-localization of visitors based on Blue-tooth. In the new scenario, researchers contribute actively in the provision of contents and social networks such as Facebook and YouTube acquire an important role not only in the distribution of contents but also in supporting the creation and development of online communities that can contribute to the collaborative production of contents [31]. In this understanding, the analysis of the organizational configurations that support the passage from Mv1 to Mv2 is not decisive. They can be considered a consequence of this new scenario in which the role of technology, researchers and visitors prevail. Therefore, the establishment of this organizational configuration composed by the focal organization and visitors and researchers communities is not central to Mv2.

From a structural perspective, the possibility to outline organizational configurations able to support innovation and provide an answer to unexpected events is fundamental. In these terms, Mv1 shows limits since a transformation is expected only within the boundaries of the focal organization. The scope of the IT artifact is to transfer contents to visitors without any impact on the overall organizational structure. Differently, in Mv2, YouTube and Facebook become part of the solution. To involve social networks entails the participation of visitors' and researchers' communities and, in this way, the platform envisages a structural arrangement in which external communities are 
connected to the focal organization. In some sense, the characteristics of Mv2 give shape to the organizational configuration.

\subsection{Midblue as an Ecogenesis}

When the strategic perspective is adopted, the concepts of dematerialization (liquification and unbundleability), rebundleability, prime mover and ecogenesis provide the lenses for understanding the IT enabled organizational transformation. The objective is to see how the Midblue project and particularly Mv1 and Mv2 transform the supply of products and services in the Midblue context (the provision of information in restricted areas). Our theoretical framework suggests to focus on the dematerialization process and on the consequent rebundleability that leads to a prime mover and to an ecogenesis.

As far as liquification concerns, that is the separation of information from the physical world, visitors have access not only to traditional sources of information such as banners, signs and flags but also to multimedia information available on their own devices equipped with a Blue-tooth connection. Mv2, in comparison with Mv1, requires a WiFi connection to access the content but, on the other hand, it encompasses the geographical limits of Mv1, which can only provide contents already owned by the focal organization. In fact, social networks in Mv2 turn out to be a source of new information and a tool for circulating them among communities of visitors and researchers.

The effects of the Midblue project on unbundleability can be subdivided according to time, space, and involving actors. Mv1 does not affect unbundleability according to time as multimedia information, once downloaded to visitors' personal devices, cannot be accessed later. Unbundleability according to space is not affected as well. It is necessary to be physically present in a determined geographical area to have access to multimedia information. Neither unbundleability according to involving actors has been touched by Mv1. The institutional arrangement or the focal organization (a zoo, a museum, a trading fair, an exhibition etc.) at the basis of the provision of these products and services have been kept unchanged.

Mv2 prompts unbundleability more deeply. In this case contents are not only available on visitors personal devices (specifically smart-phones) located in a delimited geographical area but also on social networks. Connecting the visitors and the researchers communities to the focal organization is as simple as putting a like on the Facebook page of the organization in question. This means that contents are available to visitors, researchers and their respective communities anytime (unbundleability according to time). As far as unbundleability according to space concerns, it is not necessary to be present in a determined geographical area to have access to them. The WiFi connection, the possibility to connect to the internet and then to social networks makes contents available both to visitors researchers and their communities. Mv2 envisages a significantly different organizational arrangement. The provision of contents is no more circumscribed to organizations such as zoos, museums, trading fairs, exhibitions and archaeological sites. Both visitors and researchers can acquire an active role in this respect. Visitors have the possibility to take images and videos on-site and then to post them on social networks by means of personal devices. Researchers have at disposal an extra source of information monitoring a further sample of apes. Besides, an additional channel for disseminating research results becomes available and can foster the debate within researchers' communities.

Rebundleability envisaged by the Midblue project on the basis of Mv1 consists of the possibility to provide visitors of a delimited geographical area multimedia contents related to what they are observing. A new channel of information is accessible other than typical ones as banners, signs, boards etc. With Mv2 rebundleability is more articulated. Similarly, a new channel to provide and eventually download contents to personal devices is available. However, the possibility to involve visitors and researchers gives chance to reformulate significantly products (contents) and services provided. The point now is to see if the concepts of prime mover and ecogenesis are helpful for interpreting the passage from Mv1 to Mv2. Among the attributes of a prime mover there is the capacity to foreshadow an upframing. And this is what actually takes place. Visitors and researchers are involved in the supply of contents and can create innovative channels of distribution generating a larger conception of value creation. In this understanding, the infrastructure conception comes to the fore. Mv2 can be considered as the result of the Mv1 evolution. In these cases technological aspects prevail and Mv1 can envisage a larger value system with difficulty except for the fact that a new channel of information distribution was established. However, it constituted the backbone for designing Mv2. On the other hand, Mv2 shares the characteristics of an ecogenesis as suggested by Normann [24]. In this case, a larger value system emerged as a two-phases process in which, at first, system interactions are designed and then they can evolve due to the role of visitors and researchers. This infrastructure also conceives the mobilization of traditionally dormant resources in zoos, museums, exhibitions or archaeological sites such as visitors and researchers who tend to be passive content consumers (visitors) or content producers once and for all (researchers). At the origin of Mv2 there are not power relationships but, rather, win win situations between the pivotal organization, visitors and researchers and shared rules of the game of the organizational arrangement envisaged. This suggests that an ecogenesis takes place. 


\subsection{Organizational Transformation in the Midblue Project}

The Midblue project provides an illustrative example of how strategic and structural transformations take place when IT artifacts are deployed into organizational settings. The analysis of the Mv1 and Mv2 scenarios through the lenses of our theoretical framework provides many insights in this respect. Mv1 and Mv2 are, at the same time, very similar and very different: very similar because the latter is the direct evolution of the former and a significant part of services and contents provided are the same; very different as is emerged in their analysis as a digital product [37], organizing platform [6], [8], and ecogenesis [24].

A summary of the results of this comparative analysis is presented in Table 2 in which the main findings are reported along the dimensions of our theoretical framework.

Table 2: Summary of case findings

\begin{tabular}{|l|l|l|}
\hline $\begin{array}{l}\text { PerspectivelUnit } \\
\text { of analysis }\end{array}$ & \multicolumn{1}{|c|}{ Midblue version 1 } & \multicolumn{1}{c|}{ Midblue version 2 } \\
\hline IT Architecture & $\begin{array}{l}\text { IT application based on a core set of } \\
\text { IT capabilities }\end{array}$ & $\begin{array}{l}\text { Digital platform for information sharing } \\
\text { and collaboration linked to existing } \\
\text { information infrastructures }\end{array}$ \\
\hline $\begin{array}{l}\text { Organizational } \\
\text { Structure }\end{array}$ & $\begin{array}{l}\text { Static and predefined structure } \\
\text { whose coordination mechanisms, } \\
\text { processes, and tasks are supported } \\
\text { by IT applications. }\end{array}$ & $\begin{array}{l}\text { Organization as a platform that enables a } \\
\text { multiplicity of structures and the } \\
\text { reinterpretation and reallocation of } \\
\text { resources produced and consumed within } \\
\text { visitors and researchers communities }\end{array}$ \\
\hline Business Strategy & $\begin{array}{l}\text { To enrich the experience of the } \\
\text { visitors by providing selected } \\
\text { multimedia content during their visit. }\end{array}$ & $\begin{array}{l}\text { To revise the value proposition by } \\
\text { opening the boundaries of the } \\
\text { organization to external communities of } \\
\text { customers and suppliers. The digital } \\
\text { theng } \\
\text { platform enables an ecogenesis of } \\
\text { contents and behaviours and allows to } \\
\text { encompass time and physical barriers. }\end{array}$ \\
\hline
\end{tabular}

The first perspective stresses the importance of the technological architecture in the modalities through which firms organize for innovation [37]. Mv1 and Mv2 are in fact characterized by slightly different architectures. Whereas Mv1 is an IT application based on a core set of IT capabilities (i.e. multimedia content management, localization via Bluetooth, etc.), Mv2 provides an example of a digital platform supporting a doubly distributed network. In Mv2 the IT architecture combines some additional IT capabilities (i.e. WiFi, social network interfaces) in order to build a digital platform for information sharing and collaboration linked to existing information infrastructures (i.e. Facebook, YouTube). The conversion from a digital application (Mv1) to a digital platform with a layered modular architecture (Mv2) led up to a radical change. The range of services and contents that can be provided by Mv2 is wider in comparison with Mv1. Besides, the capabilities of the former are not fully expressed yet as visitors and researchers, for example, can envisage innovative solutions in the provision of services and contents. All of this comes out plainly as Mv2 differently from Mv1 can be considered a doubly distributed network [37]. The presence of heterogeneous resources in different layers (the MidBlue application and social networks), the distribution of control of components across several businesses (the MidBlue project, YouTube, Facebook) and the fact that knowledge is shared according to several disciplines and communities (zoo, visitors and researchers) create the conditions for a promising context for the evolution of the Midblue project.

The organization as a platform [6], [8] is the second perspective taken into consideration. This perspective outlines the characteristics of a scenario able to generate continuously organizational forms based on recombination of existent resources, routines and transactions. Mv1 and Mv2 are studied according to their capacity in this recombination process for the provision of services and contents. Whereas the Mv1 assumes a static and predefined structure of coordination mechanisms, processes, and tasks, Mv2 instantiates a totally different scenario. In this case, it enables a multiplicity of structures other than the reinterpretation and reallocation of resources produced and consumed within visitors and researchers communities. The organizational architecture, routines, procedures, and frames remain stable and consistent in Mv1. In contrast, in Mv2 they are subject to innovative forces so that resources can be reinterpreted according to innovative solutions for the provision of services and contents. Besides, here the focus is posed on the dynamics at the basis of the conversion from Mv1 to Mv2. It is the result of a combination of factors that goes from the adoption of the WiFi technology for information transmission to a scenario in which visitors and researchers can become protagonists of the production and distribution of contents through social networks. Social networks acquire a further role according to the organization as a platform as factors that outline structural arrangements. YouTube and Facebook are the instruments through which visitors' and researchers' communities become part to Mv2 organizational configuration. 
Finally, dynamics of value creation are at the basis of the third perspective [24]. Here IT artifacts are seen as drivers and tools for a dematerialization process that allows to recombine the factors of production for achieving a competitive advantage. Mv1 and Mv2 are thus analyzed according to their capacity to support a dematerialization process and to exploit the potential of existing Information Infrastructures [14] in order to reconfigure the available resources available. Whereas Mv1 shows how IT applications can enrich the experience of the visitors by providing selected multimedia content during their visit, Mv2 provides an interesting example of a strategic turn enabled by a digital platform. Thanks to Mv2 the zoo can revise its value proposition by opening the boundaries of the organization to external communities of customers and suppliers. This perspective shows how digital platforms enable an ecogenesis of contents and behaviours by encompassing time and physical barriers. Liquification, unbundleability and then rebundleability are affected significantly by Mv2 but is not the same in the Mv1 case. This means that the possibility to reconfigure a specific activity such as the transmission of information to visitors of circumscribed areas (zoo, museums, exhibitions etc.) is limited in the latter. Turning to the concept of infrastructure, this limit is investigated in detail. Mv1 envisages a larger value system with difficulty as mechanisms at the basis of its evolution are not present (a two phases development process, the mobilization of dormant resources, the creation of win win situations, and the establishment of new rules of the game). In contrast, Mv2 actuates these mechanisms so that to delineate an ecogenesis [24].

These findings provide insights on the transformative power of new digital architectures. Due to the theoretical framework, different perspectives dealing with the same phenomenon. This means that both the structural level, the strategic level, and the environmental level have been investigated considering the role of digital platforms. Specifically, the Midblue case provides successfully instantiations on the modalities through which, in particular, two distinct applications such as Mv1 and Mv2 have led to a significant different transformative process. In the Mv2 casenew IT capabilities such as WiFi connections and social network APIs are at the basis of the organizational structure transformation and the implementation of new strategies.

By confirming that the architecture of digital systems can trigger organizational transformation at both structural and strategic levels, our case study provide empirical evidences that environment/strategy, strategy/structure, and strategy/IT alignments cannot be managed in traditional ways. This conclusion poses practical implications for current IT governance and IT project management practices. When dealing with digital products, practitioners should be aware on the strategic and structural implications of their choices and carefully analyze the potential of digital platforms in their context. The proposed framework can serve, for instance, as a basis for developing new conceptual tools for ex-ante and ex-post evaluation of IT projects.

\section{Conclusions}

This study has demonstrated how digital architectures, organizational structures, and business strategy are intertwined and in a state of continuous evolution. We conducted a single-case study with two embedded units of analysis in order to compare two scenarios that refer to different phases of the same Midblue project. Although the Midblue scope, objectives, and resources remained almost unchanged during the five year period of our longitudinal study, advancements in technological capabilities and information infrastructures has imposed to revise the solution scenario from Mv1 to Mv2. Different architectures led to different organizational transformation power of the IT solutions. Our theoretical framework has demonstrated to be a valid tool for analyzing these differences.

The main limitation of this study is related to the fact that the Midblue artifact has only been implemented in the pilot phase of the project. In this respect, only the design level is taken into consideration. Further, the object of the research, the provision of multimedia information to visitors of circumscribed areas, is marginal in the business environment as specific to Small and Medium Enterprises (SMEs) and then it could not be considered a representative instance.

Finally, the examination of the Midblue project according to the theoretical framework has evidenced how digital platforms can be a source of organizational transformation at both strategic and structural levels. By integrating different perspectives the central role of digital architectures in triggering and enabling organizational transformation is confirmed. Nevertheless, we emphasize also is the irresolution of the analysis even though some guiding points are placed. The scenario depicted suggests that technology, organizational arrangements and business strategies are in continuous evolution and probably Mv3 is already taking shape.

\section{References}

[1] M. Bacharach and D. Gambetta, Trust in signs, in Trust in Society (K. S. Cook, Ed.). New York: Russel Sage Foundation, 2001, pp. 148-184.

[2] P. Besson and F. Rowe, Strategizing information systems-enabled organizational transformation: A transdisciplinary review and new directions, Journal of Strategic Information Systems, vol. 21, no. 2, pp. 103124, 2012. 
[3] R. J. Boland, K. Lyytinen, and Y. Yoo, Wakes of innovation in project networks: The case of digital 3-D representations in architecture, engineering, and construction, Organization Science, vol. 18, no. 4, pp. 631647, 2007.

[4] K. E. Boulding, General systems theory-the skeleton of science, Management Science, vol. 2, no. 4, pp. 197208, 1956.

[5] P. L. Bowen, M.-Y. D. Cheung, and F. H. Rohde, Enhancing IT governance practices: A model and case study of an organization's efforts, International Journal of Accounting Information Systems, vol. 8, no. 3, pp. 191-221, 2007.

[6] C. Ciborra, The platform organization: Recombining strategies, structures, and surprises, Organization Science, vol. 7, no. 2, pp. 103-118, 1996.

[7] C. Ciborra, Teams, Markets and Systems: Business Innovation and Information Technology. Cambridge: Cambridge University Press, 1993.

[8] C. Ciborra, The Labyrinths of Information: Challenging the Wisdom of Systems. Oxford: Oxford University Press, 2002.

[9] F. Contini and G. F. Lanzara, ICT and Innovation in the Public Sector: European Studies in the Making of EGovernment. New York: Palgrave Macmillan, 2009.

[10] R. Cooper, Assemblage notes, in Organized Worlds: Explorations in Technology and Organization with Robert Cooper (R. C. H. Chia, Ed.). London; New York: Routledge, 1998, pp. 108-130.

[11] F. Fukuyama, Trust: The Social Virtues and the Creation of Prosperity. London: Hamish Hamilton, 1995.

[12] P. Gagliardi, Symbols and Artifacts: Views of the Corporate Landscape. Berlin: Walter De Gruyter, 1990.

[13] A. Ghazawneh and O. Henfridsson, Governing third-party development through platform boundary resources, in Proceedings of the International Conference on Information Systems, Saint Louis, Missouri, USA, 2010, pp. 48-48.

[14] O. Hanseth and K. Lyytinen, Design theory for dynamic complexity in information infrastructures: The case of building internet, Journal of Information Technology, vol. 25, no. 1, pp. 1-19, 2010.

[15] J. Kallinikos, The Consequences of Information: Institutional Implications of Technological Change. Cheltenham: Edward Elgar Publishing, 2006.

[16] B. Kogut, Joint ventures and the option to expand and acquire, Management Science, vol. 37, no. 1, pp. 19-33, 1991.

[17] K. Kusunoki and Y. Aoshima, Redefining innovation as system re-definition, in Dynamics of Knowledge, Corporate Systems and Innovation ( $\mathrm{H}$. Itami, K. Kusunoki, T. Numagami, and A. Takeishi, Eds.). Heidelberg: Springer, 2010, pp. 43-76.

[18] P. R. . Lawrence and J. W. Lorsch, Organization and Environment: Managing Differentiation and Integration. Cambridge: Harvard Business School Press, 1967.

[19] D. E. Leidner, J. Lo, and D. Preston, An empirical investigation of the relationship of IS strategy with firm performance, The Journal of Strategic Information Systems, vol. 20, no. 4, pp. 419-437, 2011.

[20] C. Lévi-Strauss, F. Ethnologue, and F. Ethnologist, La Pensée Sauvage. Paris: Plon, 1962.

[21] K. Lyytinen and G. Rose, The disruptive nature of information technology innovations: The case of internet computing in systems development organizations, MIS Quarterly, vol. 27, no. 4, pp. 557-595, 2003.

[22] R. E. Miles, C. C. Snow, A. D. Meyer, and H. j. jr. Coleman, Organizational strategy, structure, and process, The Academy of Management Review, vol. 3, no. 3, pp. 546-562, 1978.

[23] A. Mutti, I diffusori della fiducia, Rassegna Italiana di Sociologia, vol. 9, no. 4, pp. 553-549, 1998.

[24] R. Normann, Reframing Business: When the Map Changes the Landscape. Chichester: John Wiley \& Sons, 2001.

[25] R. Normann and R. Ramírez, From value chain to value constellation: Designing interactive strategy, Harvard Business Review, vol. 71, no. 4, pp. 65-77, 1993.

[26] R. Normann and R. Ramírez, Designing Interactive Strategy: From Value Chain to Value Constellation. Chichester: Wiley, 1994

[27] A. Ong and S. Collier, Global assemblages, anthropological problems, in Global Assemblages: technology, Politics, and Ethics as Anthropological Problems (A. Ong and S. J. Collier, Eds.). Oxford: Blackwell Publishing, 2005, pp. 3-21.

[28] A. Resca and A. D'Atri, Strategic information systems and the reconfiguration of value space: A case study of Yoox, Journal of Information, Communication and Ethics in Society, vol. 10, no. 3, pp. 131-143, 2012.

[29] E. H. Schein, Coming to a new awareness of organizational culture, The magazine-MIT Sloan Management Review, vol. 25, no. 2, pp. 2-15, 1984.

[30] P. Spagnoletti and T. Federici, Exploring the interplay between FLOSS adoption and organizational innovation, Communications of the Association for Information Systems, vol. 29, no. 15, pp. 279-298, 2011.

[31] P. Spagnoletti and A. Resca, A design theory for IT supporting online communities, in Proceedings of the $45^{\text {th }}$ Hawaii International Conference on System Sciences, Hawaii, USA, 2012, pp. 4082-4091.

[32] R. Verganti, Design-Driven Innovation: Changing the Rules of Competition by Radically Innovating What Things Mean. Boston: Harvard Business Press, 2009.

[33] G. Walsham, Interpretive case studies in IS research: Nature and method, European Journal of Information Systems, vol. 4, no. 2, pp. 74-81, 1995.

[34] G. Walsham, Doing interpretive research, European Journal of Information Systems, vol. 15, no. 3, pp. 320-330, 2006. 
[35] K. E. Weick, Organizational redesign as improvisation, in Organizational Change and Redesign: Ideas and Insights for Improving Performance (G. P. Huber and W. H. Glick, Eds.). New York: Oxford University Press, 1993, pp. 346-379.

[36] R. K. Yin, Case Study Research: Design and Methods. Thousand Oaks: Sage Publications, 2009.

[37] Y. Yoo, O. Henfridsson, and K. Lyytinen, Research commentary-The new organizing logic of digital innovation: An agenda for information systems research, Information Systems Research, vol. 21, no. 4, pp. 724-735, 2010.

[38] J. L. Zittrain, The generative internet, Harvard Law Review, vol. 119, no. 1, pp. 1974-2040, 2006. 